\title{
Aproximación a la investigación iberoamericana en educación musical
}

\section{Approach to Ibero-American Research in Music Education}

\author{
por \\ Eliton Perpetuo Rosa Pereira \\ Instituto Federal de Educação, Ciência e Tecnologia de Goiás, Brasil \\ eliton.pereira@ifg.edu.br \\ Carol Gillanders \\ Universidade de Santiago de Compostela, España \\ carol.gillanders@usc.es
}

La trayectoria histórica de la investigación en educación musical está claramente definida y estudiada en el contexto anglosajón. En España existen diversos trabajos que iluminan su recorrido y que sirven de antecedentes para la realización de un estudio exploratorio en el ámbito iberoamericano. Este trabajo aporta un acercamiento al estado de la investigación en educación musical en Iberoamérica, presentando sus principales investigadores, las revistas indexadas en las principales bases de datos y aquellas relevantes para el campo, los países con producción científica y un contexto general de la enseñanza y la investigación en educación musical en cada uno de los países que componen la comunidad iberoamericana.

Palabras clave: educación musical, Iberoamérica, investigación, líneas de investigación, producción científica.

The historical path followed by research in music education is clearly defined and studied in the Anglo-Saxon context. In Spain there are several works that illuminate its journey and that serve as background for the design of an exploratory study in the Ibero-American field. This work provides an approach to the state of research in music education in Ibero-America, presenting its main researchers, journals indexed in the main databases and those relevant to the field, countries with scientific production and a general context of education and research in music education in each one of the countries that make up the Ibero-American community.

Keywords: musical education, Ibero-America, investigation, lines of investigation, scientific production.

\section{INTRODUCCIÓN ${ }^{1}$}

Es innegable que existe una hegemonía de los países anglosajones en la investigación en educación musical, debido a la continuidad de sus instituciones culturales y a su tradición investigadora, al reconocimiento y valoración de sus publicaciones y a la disponibilidad

1 La investigación contó con apoyo financiero del Instituto Federal de Educação, Ciência e Tecnologia de Goiás (Brasil). 
de medios para ello. Encontramos detallada información en el trabajo de Mark (1992), publicado en el primer Handbook of Research on Music Teaching and Learning, una historia de la investigación educativa musical, que explica la evolución de este campo en Estados Unidos desde las primeras investigaciones, el Movimiento de Tests y Mediciones, hasta los estudios que analizan la producción científica y los temas abordados. Diez años después, Flinders y Richardson (2002) en el siguiente handbook aportan nueva información acerca del estado de la investigación cualitativa en educación musical. En el trabajo relativamente reciente de Rumpf (2012) se realiza una revisión de estudios relacionados con la educación musical del profesorado llevado a cabo en Estados Unidos entre 1982 y 2010.

La tradición investigadora en educación musical ha estado vinculada con otros campos como la psicología, antropología, etnomusicología o sociología en un intento de "adaptar y aplicar esos métodos [de investigación y constructos organizativos] al estudio de la enseñanza y el aprendizaje de la música" (Flinders y Richardson 2002: 1168). De acuerdo con Mark (1992), las primeras investigaciones pueden rastrearse hasta 1937, año en el que se envía un cuestionario al profesorado con el fin de conocer si podían cantar o tocar, entre otros temas. Hasta las primeras décadas del siglo XX, son las propias administraciones educativas las que realizan estudios similares. Continúa este autor señalando que es a partir del Movimiento de los Estudios de la Infancia y del Movimiento de la Educación Progresiva que se promueve la observación científica que genera una gran actividad investigadora en la época. Con el Movimiento de Tests y Mediciones surge un interés en dimensionar cuantitativamente las características musicales de los individuos y el talento musical y se publican varios tests o baterías, como el de Carl Emil Seashore (1866-1949), que aún siguen vigentes. Es a partir de este momento cuando los propios educadores musicales también comenzarán a interesarse por la investigación, que a la sazón estaba muy ligada al campo de la psicología. A mediados del siglo XX abundaron las investigaciones de naturaleza cuantitativa relacionadas con las aptitudes musicales, con la psicología de la música y las investigaciones históricas. Según Hanley y Montgomery (2002), la mayoría de los estudios publicados hasta la década de 1970 eran de tipo experimental o descriptivo, si bien podían encontrarse también investigaciones históricas. Es recién a partir de la década de 1980, y debido a la influencia de los trabajos de Elliot Eisner (1933-2014) y Bennett Reimer (1932-2013), cuando comienzan a publicarse trabajos de corte cualitativo. En las últimas décadas surgen centros de interés como la neurociencia (Levitin 2018), el mindfulness (Siegel), la educación somática y la salud del músico (Thayer, Brandfonbrener y Lederman 1998), la teoría crítica (Regelski 1975), los estudios culturales (Volk 1997), o los estudios de género (Green 1997).

La educación superior ha sido el espacio en donde se han realizado la mayoría de las investigaciones, generalmente por medio de tesis y trabajos de fin de máster (Mark 1992). La demanda de continuar estudios superiores al finalizar el título de grado (o profesor superior, en el caso de los conservatorios) con el fin de poder acceder a la profesión docente, comenzó, en algunos países, en la década de 1920. Desde entonces ha proliferado la oferta de másteres y de programas de doctorado (Phelps, Ferrara y Goolsby 1993). Según Reimer (2006), la influencia de la investigación en las prácticas de los docentes ha sido mínima: la mayoría de los docentes prefiere utilizar los mismos materiales y enseñar tal como aprendieron (Teachout 2012). Reimer (2006: 32), recomienda, en un esfuerzo por unir práctica e investigación, "alentar a los jóvenes estudiantes a ser investigadores (...) Es hora, sugiero, de que la misma investigación en educación musical sea sometida a un serio y disciplinado estudio respecto de su naturaleza, valor y modos de funcionamiento".

¿Qué sucede en el ámbito iberoamericano? Mediante este estudio se pretende conocer el estado de la investigación en Iberoamérica. Este artículo exploratorio forma parte de la tesis doctoral titulada "La educación musical en Brasil: temáticas, concepciones y líneas de investigación” (Pereira 2019). 


\section{ANTECEDENTES: EL CONTEXTO ESPAÑOL}

La exigencia de una formación complementaria que habilite para el ejercicio docente en la enseñanza secundaria se implanta en España a partir de 1970. Aunque en principio fue pensado como obligatorio, en la práctica se valoró como un mérito y más tarde como preceptivo si no se podía demostrar dos años de experiencia docente previa. En sus comienzos esta formación fue denominada Curso de Aptitud Pedagógica y, aunque hubo otras propuestas para sustituir este modelo, finalmente a partir de la $\mathrm{LOE}^{2}$ se especificó que los aspirantes a profesores de secundaria que desearan optar por una plaza debían cursar el Máster Universitario en Profesorado de Educación Secundaria Obligatoria y Bachillerato, Formación Profesional y Enseñanza de Lenguas (Gutiérrez González 2011).

Es interesante notar que en este nuevo modelo se introdujeron importantes modificaciones, como la inclusión de una materia que inicia al estudiantado en la investigación educativa y la obligatoriedad de presentar un trabajo final de máster. A partir de este momento surgen diferentes estudios y materiales en el área de educación musical cuyo fin fue servir de apoyo a los docentes que impartían estas nuevas materias y que, de alguna manera, han intentado cubrir un vacío en este campo, como el libro Investigación cualitativa en educación musical coordinado por Díaz Gómez y Giráldez en 2013. Sin embargo, los avances que se han iniciado en este sentido distan todavía de estar consolidados, y sigue siendo necesario aunar esfuerzos para sistematizar el conocimiento que existe de la práctica docente en el área de educación musical, ya que presenta un retraso importante respecto de la evolución en otras disciplinas. Por ejemplo, el primer texto general acerca de investigación en educación musical editado en España data de 2006 (nos referimos a la Introducción a la investigación en educación musical coordinado por la doctora Maravillas Díaz); en otros contextos el primer manual (handbook) de investigación en enseñanza y aprendizaje de la música se publicó en 1992, y en Buenos Aires se realizó una primera edición castellana del trabajo Some Approaches to Research in Music Education (Kemp 1992) en 1993.

Existen diversos motivos que pueden haber influido en la carencia de investigaciones en torno a la práctica docente musical en España, como la diferenciación entre los músicos "que piensan" (investigadores) y los músicos "que hacen" (músicos prácticos) que puede rastrearse hasta la Antigüedad (Phelps, Ferrara y Goolsby 1993). Es común pensar en los artistas como profesionales prácticos (Irwin 2008). También ha tenido un peso importante en esta situación la creación tardía de la especialidad de educación musical en las universidades y la ausencia de una tradición investigadora en los conservatorios de música (Pastor Gordero 2002). Jiménez Pérez (2015: 31) afirma que "la investigación en los conservatorios españoles ha sido escasa hasta hace pocos años y, en todo caso, ha estado generalmente vinculada a los aspectos referentes a la musicología”. Asimismo, la insuficiente preparación del profesorado en idiomas extranjeros, especialmente del inglés, dificulta que estos accedan a la literatura especializada publicada en el exterior (Rusinek 2007).

Es interesante hacer un alto aquí y recordar cómo se crearon las áreas de conocimiento en las universidades españolas y el dilema que supuso para el profesorado que impartía docencia relacionada con la música y que pudo optar por el área de adscripción. Subirats (2011) explica claramente el surgimiento del área de Didáctica de la Expresión Musical, a cuyo cargo se encuentran las materias relacionadas con la música en la educación infantil, educación primaria y el máster de secundaria.

2 Ley Orgánica 2/2006, de 3 de mayo, de Educación. BOE 4 de mayo de 206, núm.106. 
La Ley de Reforma Universitaria (1983) dispuso mediante Real Decreto 1888/1984 de 6 de septiembre de 1984, la educación musical universitaria en dos áreas de conocimiento. Una de ellas globalizada, denominada "Didáctica de la Expresión musical, plástica y corporal" y la otra "Música", no específicamente dirigida a la educación. Por acuerdo de 17 de noviembre de 1987 del Consejo de Universidades el área de "Didáctica de la Expresión musical, plástica y corporal" fue suprimida y en su lugar se crearon las áreas de "Didáctica de la Expresión Plástica" y "Didáctica de la Expresión Corporal". El profesorado de educación musical adscrito hasta entonces a este [sic] área de conocimiento suprimida sería incorporado al área de "Música". Tras la formulación de frecuentes alegaciones por parte del profesorado afectado, la Comisión Académica del Consejo de Universidades acordó el 27 de abril de 1988 crear el área de conocimiento de "Didáctica de la Expresión Musical” (DEM). (Subirats 2011: 176).

Podemos conocer la producción científica en España en el ámbito de la educación musical en varios trabajos (Narejos 2017). En estas dos últimas décadas encontramos, por ejemplo, estudios de Oriol de Alarcón (2002; 2009; 2012). En el primero, se explica la creación del área de Didáctica de la Expresión Musical en las universidades españolas a finales de los años ochenta; los estudios y tesis doctorales vinculados con el área, presentando en una tabla una relación de tesis leídas con información acerca de la universidad, título, autor y director de la tesis; la producción científica, enumerando algunos congresos y revistas del área; información relativa al profesorado numerario adscrito al área y las materias que están a su cargo; y otras actividades de extensión que realiza. Este autor identifica una "infinidad de líneas de investigación relacionadas con diferentes aspectos de la educación musical" (Oriol de Alarcón 2002: 156), exponiendo algunas de ellas. En el segundo artículo, se presenta un estudio de 366 tesis doctorales leídas entre 1998 y 2007 (cómputo por año, temas abordados y enunciados de las tesis por bloques y subclasificaciones) aportando valiosa información acerca de las investigaciones realizadas en el ámbito de la investigación musical y posibles líneas que interesaría consolidar. En cuanto al tercer artículo (2012), se detallan las diferentes líneas temáticas de las tesis doctorales leídas desde 1978 hasta 2011, identificando 620 tesis durante este período que pueden agruparse en 25 líneas diferentes. Es interesante notar que durante un período menor (desde 1985 a 1995), en el ámbito de las Bellas Artes se leyeron 683 tesis (Marín Viadel, Tolosa Marin y Laiglesia 1998).

Hay también otros trabajos centrados en alguna región específica, como la investigación de Balerdi (2007), la que presenta una relación de tesis doctorales realizadas en las universidades del País Vasco y de Navarra, así como en otras universidades extranjeras cuando estas están relacionadas con la música vasca. En total corresponden a 135 tesis defendidas entre 1954 y 2006 , y 15 en proceso de elaboración. Por otra parte, encontramos breves referencias a varios centros de interés relacionados con la educación musical que atraen a los investigadores en España en algún Handbook internacional, como es el caso de la aportación de Rusinek (2007) referido a la creatividad en educación musical.

De manera similar, el trabajo de Abril y Abril (2017) analiza la situación de la educación musical en las Américas. Desde una perspectiva socioecológica, los autores presentan una síntesis de estudios que describen la situación de la educación musical en el contexto escolar en varios países, tanto en los países de América del Norte como de América Latina.

\section{METODOLOGÍA}

Con el fin de conocer la producción científica relacionada con la educación musical en el contexto iberoamericano se ha llevado a cabo un estudio exploratorio. De acuerdo con Hernández Sampieri, Fernández-Collado y Baptista Lucio, este tipo de estudio se realiza "cuando el objetivo es examinar un tema o problema de investigación poco estudiado, del 
cual se tienen muchas dudas o no se ha elaborado antes" (2013: 100). Desde el campo de la investigación educativa, se toman como base las directrices metodológicas de los trabajos de Goergen (1991) y Bray y Adamson (2010), mientras que desde el área de educación musical se tienen en cuenta otras investigaciones similares como los trabajos de Cateura Mateu (1992), Cykler (1969), Fernandes (2014) y Kemp y Lepherd (1992).

El objetivo de esta investigación exploratoria es conocer la investigación en educación musical llevada a cabo en el ámbito iberoamericano, realizando una aproximación a la situación de la enseñanza musical en cada uno de los países que lo integran ${ }^{3}$. Según la Real Academia Española (2018: §1), Iberoamérica es el término que designa "el conjunto de países americanos que formaron parte de los reinos de España y Portugal [...] iberoamericano, se refiere normalmente solo a lo perteneciente o relativo a Iberoamérica, esto es, a los países americanos de lengua española y portuguesa”. Nuestra lista no incluye países colonizados por España y Portugal en África y Asia.

Podemos decir que la investigación en educación musical sufre una influencia significativa del modo en que la sociedad vive, comprende y valora la música en su estructura educativa y cotidiana. Por ello, en esta investigación se estudian: 1) los investigadores; 2) las revistas científicas; 3) los países iberoamericanos con producción científica en educación musical; 4) el contexto general de la enseñanza y la investigación en música en cada país. Debido a la extensión del estudio, la información que se presenta no tiene en cuenta el factor histórico, tipos de producción realizados, ni tampoco se caracteriza a los autores.

En el primer caso, las búsquedas se realizaron por medio del análisis de los nombres de los profesores universitarios, miembros de las asociaciones de cada país, miembros de comités de revistas científicas del área y por una búsqueda en línea, teniendo como base principalmente las publicaciones listadas en Google Académico, considerando de esta forma la identificación de la producción científica y académica disponible y abierta en línea de cada investigador o investigadora. En relación con las revistas científicas, se ha recurrido a MIAR (Matriz de Información para el Análisis de Revistas) de la Facultad de Biblioteconomía y Documentación de la Universidad de Barcelona, cotejando la información facilitada por las propias revistas y por las bases de datos estudiadas.

Para realizar un análisis descriptivo de la situación de la educación musical en el contexto iberoamericano por países, fue necesario identificar y clasificar la producción científica al respecto. En primer lugar, se tuvo en consideración los países con doctorado en Educación Musical, Música, Arte o Educación, considerando que la investigación puede darse principalmente en esas áreas. En segundo lugar, se tuvieron en cuenta las asociaciones y revistas científicas que publican artículos con la temática de educación musical. Se accedió a la Web of Science y a SCImago Journal Rank (SJR). Posteriormente, se revisaron las webs de las universidades para confirmar la producción científica realizada en esos países, aunque no siempre están detalladas las publicaciones. Se realizó una búsqueda en línea con el descriptor "educación musical" y términos relacionados. Debemos de tener en cuenta, de acuerdo con Vélez-Cuartas, Lucio-Arias y Leydesdorff (2015: 36), que el desarrollo de la investigación en América Latina "se enfrenta a múltiples desafíos, incluyendo presupuestos limitados en I+D, baja participación de los investigadores y personal cualificado en el trabajo,

3 Debe tenerse en cuenta la asimetría existente entre la formación pedagógica y la investigadora en los diversos países estudiados, que influyen de forma determinante en el desarrollo de la educación musical en Iberoamérica. Asimismo, debe considerarse la realidad de la educación musical escolar, la formación del profesorado y las políticas públicas en materia de educación que poseen un carácter dinámico y mudable. 
infraestructura y laboratorios especializados insuficientes, y una inadecuada circulación y visibilización de los resultados de investigación".

\section{RESULTADOS}

Con relación a los educadores musicales e investigadores del área, teniendo en cuenta la producción científica o académica y la participación en eventos como oradores y como representantes de organizaciones, identificamos exponentes en cada uno de los países (independientemente del país de origen), como se muestra en la Tabla 1 (si bien este trabajo no tiene la intención de revelar la totalidad de investigadores e investigadoras del área). De ellos, casi todos tienen producción académica o científica publicada y accesible en línea (ver Tabla 1).

\section{TABLA 1: EDUCADORES MUSICALES IBEROAMERICANOS CON PRODUCCIÓN ACADÉMICA}

\begin{tabular}{|c|c|c|c|}
\hline Argentina & Chile & España & México \\
\hline $\begin{array}{c}\text { Bas, Analía } \\
\text { Carabetta, Silvia } \\
\text { Couve, Alicia C. de } \\
\text { Dal Pino, Claudia } \\
\text { De Vicenzi, Alejandro } \\
\text { Dutto, Susana } \\
\text { Espinosa, Susana } \\
\text { Frega, Ana Lucía } \\
\text { González Marti, Ángel } \\
\text { Grãtzer, Dina Poch de } \\
\text { Hemsy de Gainza, } \\
\text { Violeta } \\
\text { Malbrán, Silvia } \\
\text { Martínez, Isabel } \\
\text { Cecilia } \\
\text { Shapiro, Alicia } \\
\text { Simonovich, } \\
\text { Alejandro }\end{array}$ & $\begin{array}{c}\text { Bindhoff de Sigren, } \\
\text { Cora } \\
\text { Candia, Sergio } \\
\text { Cartes, Brunilda } \\
\text { Matthey Correa, } \\
\text { Gabriel } \\
\text { Cortés, Renán } \\
\text { Fernández Grez, } \\
\text { Margarita } \\
\text { Gayán, Elisa } \\
\text { Isamitt, Carlos } \\
\text { Kock, Hermann } \\
\text { Merino Montero, Luis } \\
\text { Poblete Lagos, Carlos } \\
\text { Pierret, Florencia } \\
\text { Reyes, Laura } \\
\text { Saavedra, María } \\
\text { Eugenia } \\
\text { Santa Cruz, Domingo }\end{array}$ & $\begin{array}{c}\text { Alsina Masmitjà, Pep } \\
\text { Aramberri, Ma José } \\
\text { Arnaus, Ängels } \\
\text { Aróstegui Plaza, José } \\
\text { Luis } \\
\text { Arriaga Sanz, Cristina } \\
\text { Díaz Gómez, } \\
\text { Maravillas } \\
\text { Giráldez Hayes, } \\
\text { Andrea } \\
\text { Muñoz Muñoz, Juan } \\
\text { R. } \\
\text { Jimeno Gracia, Ma } \\
\text { Manuela } \\
\text { Jorquera Jaramillo, } \\
\text { Ma Cecília } \\
\text { Lago Castro, Pilar } \\
\text { Loizaga Cano, Maria } \\
\text { Ibarretxe Txakartegi, } \\
\text { Gotzon } \\
\text { Oriol de Alarcon, } \\
\text { Nicolás } \\
\text { Pliego de Andrés, } \\
\text { Víctor } \\
\text { Sarfson Gleizer, } \\
\text { Susana } \\
\text { Subirats Bayego, Ma } \\
\text { dels Ângels }\end{array}$ & $\begin{array}{c}\text { Ángel Sozio, Juan } \\
\text { Alfonso Estrada, Luis } \\
\text { Castro-Sierra, } \\
\text { Eduardo } \\
\text { Dultzin Dubin, } \\
\text { Susana } \\
\text { Fernández, Aldara } \\
\text { González-Moreno, } \\
\text { Patrícia A. } \\
\text { Gutiérrez Sandoval, } \\
\text { Pavel R. } \\
\text { Martínez Rivera, } \\
\text { Víctor S. } \\
\text { Martínez Salgado, } \\
\text { Graciela Guadalupe } \\
\text { Pérez Expósito, } \\
\text { Leonel } \\
\text { Reynoso Vargas, Karla } \\
\text { M. } \\
\text { Robledo Barros, Rosa } \\
\text { Roubina Milner, } \\
\text { Evguenia } \\
\text { Suárez, Jorge } \\
\text { Toriz Sandoval, Juan } \\
\text { Rafael } \\
\text { Valenzuela Remolina, } \\
\text { Miguel } \\
\text { Viveros Castañeda, } \\
\text { Susana }\end{array}$ \\
\hline
\end{tabular}




\begin{tabular}{|c|c|c|c|}
\hline Portugal & Colombia & Cuba & Brasil \\
\hline $\begin{array}{c}\text { Alves, Vasco } \\
\text { Artiaga, Maria José } \\
\text { Bessa, Rui } \\
\text { Boal-Palheiros, Graça } \\
\text { Boia, Pedro Santos } \\
\text { Costa, Jorge } \\
\text { Alexandre } \\
\text { Foleto, Clarissa } \\
\text { Godinho, José Carlos } \\
\text { Gonçalves, Carlos } \\
\text { Lopes, Filipe } \\
\text { Mota, Graça } \\
\text { Neves, José António } \\
\text { Santos, Margarida F. } \\
\text { Vasconcelos, António } \\
\text { Â. } \\
\text { Veloso, Ana Luísa }\end{array}$ & $\begin{array}{c}\text { Barriga Monroy, } \\
\text { Martha } \\
\text { Casas Fernández, } \\
\text { Patricia } \\
\text { López Gil, Gustavo A. } \\
\text { Morales Ortiz, Blanca } \\
\text { L. } \\
\text { Ochoa Escobar, Juan } \\
\text { S. } \\
\text { Perez Herrera, } \\
\text { Manuel A. } \\
\text { Restrepo, Clara } \\
\text { Teresa } \\
\text { Rodríguez Melo, } \\
\text { Martha E. } \\
\text { Samper Arbeláez, } \\
\text { Andrés } \\
\text { Valencia Mendoza, } \\
\text { Gloria }\end{array}$ & $\begin{array}{c}\text { Araúz Batista, Aleyvis } \\
\text { Borlot Faure, Aysmara } \\
\text { Galán Rizo, Radisbel } \\
\text { Jaime Infante, Yesenia } \\
\text { Lavielle Pullés, Ligia } \\
\text { Sánchez Ortega, } \\
\text { Paula } \\
\text { Sánchez Valle, Niurka } \\
\text { M. }\end{array}$ & $\begin{array}{c}\text { Arroyo, Margarete } \\
\text { Barbosa, Joel Luis } \\
\text { Beineke, Viviane } \\
\text { Bellochio, Claudia } \\
\text { Ribeiro } \\
\text { Beyer, Esther } \\
\text { Brito, Teca Alencar de } \\
\text { Del-Ben, Luciana } \\
\text { Fernandes, José } \\
\text { Nunes } \\
\text { Figueiredo, Sergio } \\
\text { L. F. } \\
\text { Fonterrada, Marisa } \\
\text { T.de O. } \\
\text { França, Cecilia } \\
\text { Cavalieri } \\
\text { Fucci-Amato, Rita } \\
\text { de C. } \\
\text { Fuks, Rosa }\end{array}$ \\
\hline Costa Rica & Ecuador & Guatemala & Wilke F. \\
\hline $\begin{array}{c}\text { Deras Carrillo, Carlos } \\
\text { Méndez Navas, } \\
\text { Carmen María }\end{array}$ & $\begin{array}{l}\text { Pinza, Benjamín } \\
\text { Narváez Iñiguez, Iván } \\
\text { Antonio }\end{array}$ & $\begin{array}{l}\text { Batres Moreno, Ethel } \\
\text { Pérez, Juan Carlos }\end{array}$ & $\begin{array}{c}\text { Souza } \\
\text { Hentschke, Liane } \\
\text { Joly, Ilza Zenker Leme }\end{array}$ \\
\hline Perú & Uruguay & Venezuela & Kleber, Magali \\
\hline $\begin{array}{l}\text { Elías Llanos, Carlos } \\
\text { Fernando } \\
\text { Lloréns Amico, José } \\
\text { Antonio } \\
\text { Romero Soto, Lilia }\end{array}$ & $\begin{array}{l}\text { Soto, Adriana } \\
\text { Tejera, Andrea }\end{array}$ & $\begin{array}{l}\text { Rodríguez, Jesús } \\
\text { Sánchez, Freddy }\end{array}$ & $\begin{array}{c}\text { Lima, Sônia Albano } \\
\text { de } \\
\text { Mateiro, Teresa } \\
\text { Penna, Maura } \\
\text { Oliveira, Alda de Jesus } \\
\text { Queiroz, Luís Ricardo } \\
\text { S. } \\
\text { Requião, Luciana } \\
\text { Santos, Regina Marcia } \\
\text { S. } \\
\text { Souza, Jusamara } \\
\text { Tourinho, Cristina } \\
\text { Tourinho, Irene }\end{array}$ \\
\hline
\end{tabular}

Fuente: Elaboración propia.

En la Tabla 2 se presentan las principales revistas científicas de los países iberoamericanos indexadas en Scopus, Erihplus o ESCI (activas e inactivas) de las áreas "Música" y 
"Educación Musical”, con acceso en línea. Al igual que sucede con otras áreas pertenecientes a las Ciencias Sociales, estas no están representadas en el Journal Citation Report (JCR). Señala Porta (2014: 608) que en España "no existe ninguna revista específica de educación musical ni de música considerada de alto impacto, es decir en el JCR o en el primer cuartil de los indicadores de calidad de las revistas españolas". A esta realidad, tal como puede verse en la Tabla 2, se suman las publicaciones de los demás países iberoamericanos (ver Tabla 2).

TABLA. 2: REVISTAS E INDEXACIÓN

\begin{tabular}{|c|c|c|c|c|}
\hline Revistas & JCR & SCOPUS & $\begin{array}{l}\text { ERIH/ } \\
\text { ERIH } \\
\text { PLUS }\end{array}$ & ESCI \\
\hline $\begin{array}{l}\text { Anuario Musical } \\
\text { (ISSN 1988-4125) }\end{array}$ & & $\mathrm{X}$ & $\mathrm{x}$ & $\mathrm{x}$ \\
\hline $\begin{array}{l}\text { Cuadernos de música, artes visuales y artes escénicas } \\
\text { (ISSN 2215-9959) }\end{array}$ & & $\mathrm{X}$ & & $\mathrm{x}$ \\
\hline $\begin{array}{l}\text { El oido pensante } \\
\text { (ISSN 2250-7116) }\end{array}$ & & $\mathrm{X}$ & $\mathrm{x}$ & \\
\hline $\begin{array}{c}\text { Musica Hodie } \\
\text { (ISSN 2317-6776) }\end{array}$ & & $\mathrm{x}$ & & \\
\hline $\begin{array}{l}\text { Opus - Revista electrónica da Anppom } \\
\text { (ISSN 1517-7017) }\end{array}$ & & $\mathrm{X}$ & & \\
\hline $\begin{array}{l}\text { Revista de Musicología } \\
\text { (ISSN 0210-1459) }\end{array}$ & & $\mathrm{X}$ & $\mathrm{x}$ & $\mathrm{x}$ \\
\hline $\begin{array}{c}\text { Revista Electrónica Complutense de Investigación } \\
\text { en Educación Musical } \\
\text { (ISSN 1698-7454) }\end{array}$ & & $\mathrm{X}$ & $\mathrm{x}$ & $\mathrm{x}$ \\
\hline $\begin{array}{l}\text { Revista Electrónica de LEEME } \\
\text { (ISSN 1575-9563) }\end{array}$ & & $\mathrm{X}$ & $\mathrm{x}$ & $\mathrm{x}$ \\
\hline $\begin{array}{l}\text { Revista Internacional de Educación Musical } \\
\text { (ISSN 2307-4841) }\end{array}$ & & & $\mathrm{x}$ & $\mathrm{x}$ \\
\hline $\begin{array}{l}\text { Revista Musical Chilena } \\
\quad \text { (ISSN 0716-2790) }\end{array}$ & & $\mathrm{X}$ & & \\
\hline $\begin{array}{l}\text { Revista Per Musi } \\
\text { (ISSN 2317-6377) }\end{array}$ & & $\mathrm{X}$ & & \\
\hline $\begin{array}{l}\text { Revista Portuguesa de Musicología } \\
\text { (ISSN 0871-9705) }\end{array}$ & & & $\mathrm{x}$ & \\
\hline
\end{tabular}




\begin{tabular}{|c|c|c|c|c|}
\hline Revistas & JCR & SCOPUS & $\begin{array}{c}\text { ERIH/ } \\
\text { ERIH } \\
\text { PLUS }\end{array}$ & ESCI \\
\hline $\begin{array}{c}\text { Revista Resonancias } \\
\text { (ISSN 0719-5702) }\end{array}$ & & $\mathrm{X}$ & & \\
\hline $\begin{array}{c}\text { Trans: Revista Transcultural de Música } \\
\text { (ISSN 1697-0101) }\end{array}$ & & & $\mathrm{x}$ & $\mathrm{x}$ \\
\hline
\end{tabular}

Fuente: Elaboración propia.

Otras revistas de las áreas mencionadas no indexadas en los anteriores listados son las siguientes (excluimos las que no tienen ISSN):

- Arte y Movimiento (ISSN 1989-9548)

- Cuadernos de investigación musical (ISSN 2530-6847)

- Enseñar Música - Revista Panamericana de Investigación

(ISSN 2314-2847). El dominio expiró 22/09/2018.

- Eufonía (ISSN 2014-4741)

- Música, cultura y pensamiento (ISSN 2145-4728)

- Música y educación (ISSN 0214-4786).

Último número disponible, diciembre 2014.

- MusMat-Revista brasileira de música y matemática (ISSN 2526-3757)

- Neuma-Revista de música y docencia musical (ISSN 0718-7017)

- Perspectivas Interdisciplinaria de Música (ISSN 1870-5758)

Último número 2010.

- Revista Brasileira de Música (ISSN 0103-7595)

Último número disponible 2016.

- Revista foro de educación musical, artes y pedagogía (eISSN 2525-1317)

- Revista Heterofonía (ISSN 0018-1137)

Último número disponible 2007.

- Revista Ricercare (ISSN 2346-4879)

- Revista da ABEM (ISSN 1518-2630 e 2358-033X)

En la Tabla 3 se presentan los diferentes países iberoamericanos organizados según el volumen de su producción científica relacionada con la educación musical (ver Tabla 3).

TABLA. 3: CLASIFICACIÓN DE LOS PAÍSES IBEROAMERICANOS SEGÚN SU PRODUCGIÓN CIENTÍFICA EN EDUCACIÓN MUSICAL

\begin{tabular}{|c|c|c|c|}
\hline Grupo 1 & Grupo 2 & Grupo 3 & Grupo 4 \\
\hline Argentina & Colombia & Bolivia & El Salvador \\
\hline Brasil & Cuba & Costa Rica & Guatemala \\
\hline
\end{tabular}




\begin{tabular}{|c|c|c|c|}
\hline Grupo 1 & Grupo 2 & Grupo 3 & Grupo 4 \\
\hline Chile & Perú & Ecuador & Honduras \\
\hline España & Venezuela & Nicaragua & Panamá \\
\hline México & - & Paraguay & República Dominicana \\
\hline Portugal & - & Puerto Rico & Uruguay \\
\hline
\end{tabular}

Fuente: Elaboración propia.

El grupo 1 presenta países con producción científica en educación musical significativa. Podemos encontrar tesis doctorales y artículos científicos publicados y accesibles en línea, y estos son los únicos países iberoamericanos hasta 2016 con posgrados que admiten la investigación en educación musical.

En el grupo 2 se encuentra un conjunto de países con una producción científica en educación musical de difícil acceso, a pesar de presentar otra producción en el área y de ofrecer bachilleratos y licenciaturas en música, así como maestrías y doctorados en educación o en música y musicología.

En cuanto al tercer grupo, son países que tienen potencial de producción doctoral a medio plazo. Tienen programas recientes de licenciatura en música, pero en la búsqueda realizada no se encontraron tesis de doctorado con la temática en educación musical, a pesar de que en algunos casos presentan cursos de doctorado en educación y revistas del área de musicología. Aunque en estos países es posible encontrar artículos que relacionan música y educación, la búsqueda no es tan fácil y con resultados seguros en relación con textos oriundos de investigaciones académicas.

El grupo 4 no posee producción científica en educación musical accesible en línea. Se presenta una menor perspectiva de producción en este ámbito a corto plazo por la expansión de titulaciones en sus centros, lo que nos lleva a pensar que será solamente a largo plazo cuando se revierta esta situación. En algunos de esos países el contenido relacionado con la música se vincula con los programas curriculares de la educación básica, pero la mayoría no posee cursos de formación superior en música o licenciatura en música.

En relación con los países que conforman el grupo 1 de la Tabla 3, en Argentina encontramos una gran cantidad de producción científica y académica en educación musical, siendo publicada en revistas específicas del área, así como de educación. La enseñanza de música en la red de educación básica es fuerte en su concepción y existe un gran volumen de cursos de licenciatura, maestrías y doctorados dirigidos al área. Destacan dos grandes nombres: Ana Lucía Frega (n. 1935) y Violeta Hemsy de Gainza (n. 1929). Son grandes figuras de la educación musical, la primera por su intensa producción científica y la segunda, aun no siendo doctora, por la profunda reflexión que realiza acerca de la pedagogía musical en diferentes ámbitos, al mismo tiempo que propone una síntesis por medio de sus diferentes materiales pedagógicos que finalmente desemboca en su propuesta de la Pedagogía Musical Abierta, a la vez que por su representatividad e influencia entre los países latinos, principalmente por la creación del Foro Latinoamericano de Educación Musical (FLADEM).

En Brasil la educación musical se desarrolló significativamente a partir de la década de 1980 , tanto en el contexto escolar como en el contexto de la investigación. Si consultamos la web del Ministerio de Educación de Brasil (MEC) es posible verificar la existencia de más de cien instituciones que ofrecen cursos superiores en música con reconocimiento 
nacional oficial. De modo análogo, las licenciaturas y los bachilleratos en música en Brasil suman cerca de cien cursos para cada modalidad, además de cientos de cursos técnicos y profesionalizadores en un instrumento musical. Se calcula que hay en el país más de 18.000 educadores musicales ejerciendo la profesión en varios contextos ${ }^{4}$. De modo análogo, se constata que el posgrado en Educación y Música también tuvo un crecimiento a partir del año 2000 repercutiendo en la producción científica y fortaleciendo la educación musical en el contexto académico y científico. En la actualidad se ofrecen 19 cursos de maestría en música y 10 doctorados en el área. La investigación en educación musical también se desarrolla en los más de 200 programas de posgrado en educación.

La educación musical en Chile se hace presente en la educación pública básica en todas las fases. Las numerosas facultades de educación ofrecen licenciaturas en música y los conservatorios y escuelas de música se concentran más en la enseñanza específica ${ }^{5}$. Poblete Lagos (2010), investigador y educador musical chileno, hace un análisis de los currículos oficiales de la educación musical en Chile, de 1965, 1981 y 1996-1998. Este último, aun parcialmente en vigor ${ }^{6}$, presenta la educación artística como obligatoria en las distintas fases de la educación básica, con dos o cuatro horas de disciplina por semana, y en este contexto la música puede ser una opción de la escuela, la que compite con artes plásticas y artes escénicas. Según Margaño et al. (1961), el primer congreso de educación musical chileno fue en 1958, lo que muestra la tradición de las organizaciones de los profesores de música del país. Aróstegui (2010) y aún Burgos Peredo, Perales Palacios y Gutiérrez Pérez (2010) presentan algunos aspectos indicadores de un progreso en la calidad formativa de los maestros de música en el país.

En cuanto a España, la educación musical se hace presente en todos los niveles de la educación básica, aunque existe la posibilidad de la optatividad a partir de la última reforma. A pesar de la baja carga horaria para el contenido musical en la escuela, el profesorado tiene acceso a una formación pública y especializada en el área. De la misma manera que la formación de profesores se da en el contexto de las facultades de formación del profesorado, también la investigación en educación musical se encuentra casi exclusivamente en los centros de posgrado en el campo de la educación, aunque lentamente este panorama está cambiando. Oriol de Alarcón (2007) destaca algunos nombres de educadores musicales españoles que difundieron a partir de mediados del siglo XX la enseñanza musical en la formación general. Entre los educadores musicales pioneros en este país encontramos a Joan Llongueras i Badía, Irineu Segarra i Malla, María Rosa Font Fuster, Montserrat Sanuy Simón, Luis Elizalde Ochoa y María de los Ángeles Cosculluela Mazcaray. Si bien puede decirse que no tiene la repercusión de los países anglosajones, presenta a lo largo de la historia varios proyectos y acciones ejemplares de pedagogos, músicos, compositores y artistas que colaboraron para que la educación musical ganara el actual estatus en la educación básica del país.

A diferencia de otros países iberoamericanos, los conservatorios de música en España no se incorporaron a las universidades, de manera que la investigación doctoral se lleva a cabo en las universidades, ofertándose doctorados en musicología o la posibilidad de

4 Instituto Nacional de Estudios e Investigaciones Educativas Anísio Teixeira (INEP-Brasil). Considerando las Sinopsis Estadísticas de la Educación Superior - Graduación (de 1995 a 2015). Disponible en: http://inep.gov.br/web/guest/sinopses-estatisticas-da-educacao-superior [acceso: 12 de junio, 2017].

5 Los trabajos de Poblete Lagos (2017) y Poblete Lagos et al. (2019) proporcionan contexto y permiten ampliar la información acerca de la situación de la educación musical en el país.

$6 \mathrm{Al}$ respecto ver página del área de Currículum del Ministerio de Educación de Chile: Disponible en: https://www.curriculumnacional.cl/614/w3-propertyvalue-52051.html [acceso: 12 de junio, 2017]. 
realizar doctorados en las facultades de educación. A base de un análisis de los trabajos de Oriol de Alarcón (2002; 2009; 2012), Gillanders y Martínez Casillas (2005) y Narejos (2017) es posible comprender que la investigación en música y en educación musical es muy amplia en este país. Los investigadores, citados en la Tabla 1 anteriormente presentada, desarrollan estudios en torno a diversas temáticas. En comparación con el resto de los países, tanto en el aspecto cuantitativo como en los aspectos de tradición y amplitud de temáticas tratadas en los trabajos, la investigación en educación musical en España se asemeja más a la producción internacional anglosajona.

México presenta una relativa cantidad de tesis doctorales y artículos científicos del área de la educación musical. Las escuelas de música y conservatorios pasaron por procesos de evaluación externa y recibieron orientaciones internacionales para mejorar su enseñanza. El gobierno publicó textos orientativos a los profesores acerca de la enseñanza de música en la educación básica, cuyos textos abarcan desde el primero al sexto año y el secundario (México 2011a, 2011b). Asimismo, la enseñanza de música en la educación básica tiene muchas carencias y la formación de profesorado está comenzando con la implantación de algunas licenciaturas en música en el país. La producción científica que discute la educación musical se encuentra principalmente en el posgrado en educación. No obstante, algunos de los conservatorios de música también poseen investigaciones musicológicas y algunos están vinculados con las universidades, ofreciendo cursos de bachillerato y licenciatura en música y, en algunos casos, cursos de posgrado, maestría y doctorado en música.

En Larrañaga Torróntegui (2016), Luna Ortiz (2008) y Martínez Rivera (2016) es posible encontrar información acerca de la presencia de la educación musical en la escuela mexicana. La educación musical en México, con relación a la presencia de la enseñanza de música en la educación básica, es muy similar a la de Brasil, Argentina y Chile. Hay varios cursos de formación de profesorado de música, pero la profesión de profesor se enfrenta a una seria devaluación social. A pesar de que las escuelas, en general, no presentan una estructura suficiente para atender las necesidades de una clase de música y hay carencias en la formación del profesorado, destacan iniciativas educativo-musicales como las orquestas infantiles y juveniles que replican el modelo del Sistema Venezolano (Capistrán Gracia 2021). Hay muchos profesores y músicos que trabajan en la enseñanza de la música en las escuelas, pero sin formación superior en el área, principalmente debido al crecimiento de las escuelas de tiempo integral (escuelas de tiempo completo).

De acuerdo con Mota (2014), la educación musical en Portugal se hace presente en casi toda la educación básica, especialmente en los años previos a la secundaria. Sin embargo, debido al enfoque curricular que revalorizó las disciplinas del portugués y matemáticas, las políticas públicas no han prestado atención a esta área, por lo que la educación musical está presente solo en las escuelas donde hay un profesor con formación; es decir, esta asignatura está presente en el marco de competencias esenciales del currículo nacional de la educación básica, pero en la práctica no se cumple. A pesar de esta dura realidad, la educación musical ha crecido mucho en el país. La formación del profesorado, las organizaciones y las publicaciones han posibilitado un mayor número de adeptos en las últimas décadas. En el contexto de la investigación en educación musical, la mayoría de las producciones se encuentran en el posgrado en educación. Según Palheiros (1999), el desarrollo de la investigación en Portugal se debe a varios factores, incluyendo:

(...) la expansión de la educación musical, en particular el desarrollo de la educación musical en el sistema educativo portugués, la creación de cursos de música y la educación musical a nivel de la universidad politécnica y la educación superior, la educación continua de los profesores en metodologías de la Educación Musical y la formación especializada de docentes en estudios de postgrado (Palheiros 1999: 15). 
Por tanto, la educación musical en el contexto de la educación básica en Portugal es similar al sistema español y al brasileño. Aun así, ambos países de habla portuguesa siguen por detrás de España respecto de la presencia de la educación musical en las escuelas ordinarias y en relación con la producción científica en educación musical. En este caso España se encuentra al frente de los demás países iberoamericanos.

Según Rodríguez Melo (2015), la educación musical en Colombia vive una contradicción entre los ámbitos legales y la práctica escolar, pues las orientaciones del Ministerio de Educación Nacional del país enumeran muchos contenidos y competencias para el currículo, pero la formación de los profesores de música aún es muy carente en el país. Si por un lado hay leyes y orientaciones para la enseñanza, por otro lado, no hay compromiso con el fomento o formación del profesorado e implantación de la educación musical en la educación básica, que configura el contexto de la precariedad general de la educación en el país. Según Rodríguez Melo (2015: 7), "actualmente existen 13 licenciaturas en música en el país, la mayoría situada en ciudades capitales de la región andina”. Las temáticas más tratadas en los trabajos de fin de curso en la formación inicial del profesorado de música colombiano, según un estudio desde el 2000 al 2010, son: "Técnica instrumental, Teoría y educación musical, Investigación en músicas tradicionales, Integración con otras artes, la obra de autores, la producción, el uso de las TIC7 y la gestión" (Rodríguez Melo 2015: 8).

Cuba tiene doctorado en educación y en artes con línea de investigación en música y algunos bachilleratos y licenciaturas en música. Las revistas presentan, como las demás revistas de los países de América Central, una cantidad muy pequeña de artículos con temática orientada a la educación musical. Hay algunos textos que abordan la temática, pero no son oriundos de estudios académicos sistematizados, provenientes de disertaciones, tesis o de investigaciones desarrolladas por grupos de investigación, o incluso proyectos individuales de académicos de las universidades. Cerca de diez artículos encontrados abordan la temática de forma científica, junto con una cantidad pequeña de tesis doctorales en torno a la educación musical.

En Perú hay varios cursos de doctorado en educación, pero las tesis son muy diversificadas y no se han encontrado tesis con un foco en la enseñanza-aprendizaje musical. La educación musical no está presente en la red de educación básica de forma sistemática, pero las escuelas de música y conservatorios son tradicionales en el trabajo con la enseñanza específica de instrumento y canto. Los repositorios consultados indican una notoria tendencia a la ampliación de los cursos de formación de profesores de música en cursos superiores de licenciatura.

En Venezuela, según Bordones (2013), la condición de la educación musical, en cuanto a la presencia en la escuela de educación básica y en lo que se refiere a la investigación, es similar al resto de países del grupo 2, Colombia, Cuba y Perú. A pesar de haber escuelas de música, proyectos de enseñanza específicos de iniciación musical, además de los conservatorios, maestrías y doctorados en educación, así como la mayoría de los países de los grupos 3 y 4, Venezuela no presenta producción científica fácilmente accesible en educación musical a nivel de postgrado, como es posible confirmar al consultar los enlaces de los servicios públicos y privados del área. Al igual que en los demás países de este grupo, hay una patente ampliación de la formación de profesores de música por medio de cursos superiores de licenciatura.

En Uruguay, las bibliotecas ofrecen búsquedas en bases de datos y repositorios españoles y en repositorios vinculados a otras universidades iberoamericanas. Hay dos doctorados y varias maestrías en educación. No encontramos ninguna maestría o doctorado en música

7 Tecnologías de la Información y de la Comunicación. 
hasta 2016, lo que ocurre también en todos los países del grupo 3. Hay tres licenciaturas en música y una maestría en musicología, pero no hemos encontrado producción doctoral en educación musical aunque sí existen diferentes trabajos finales de máster relacionados con el tema. Además, es muy destacada la labor realizada por el compositor y musicólogo Coriún Aharonián (1940-2017) que también ha publicado escritos vinculados a la educación musical.

Y esta condición es una curiosa característica de la investigación en educación musical, pues la mayoría de los estudios de posgrado son desarrollados en América Latina en los programas de educación, música o artes. En este sentido, la articulación social y cultural entre los campos de la educación y la música, y la relación entre las políticas de democratización de la enseñanza de la música y la producción de conocimiento en educación musical parecen estar profundamente ligadas. Es decir, para que haya interés en la investigación, es imperativa la concepción de democratización de la enseñanza musical sumado a una apertura de los programas de postgrado para la investigación en el área, lo que es recursivo para el fortalecimiento de la presencia de la educación musical en la educación formal.

De la mayoría de los países del grupo 3, Bolivia, Panamá, Puerto Rico, Costa Rica ${ }^{8}$ y Ecuador, no hemos encontrado producción científica doctoral accesible en línea acerca de educación musical a pesar de tener una licenciatura en música. Sin embargo, encontramos personalidades del ámbito que sí son doctores, por ejemplo, en cultura artística centroamericana. Algunos países del grupo 2, como Cuba, Colombia y Venezuela tienen doctorado en educación, pero la producción científica en la temática de educación musical no es frecuente en tesis doctorales, y resulta poco accesible en línea.

Podemos decir que solo diez de los países iberoamericanos tienen producción científica accesible en línea en el campo de la educación musical, teniendo en cuenta aquí las tesis doctorales y artículos científicos: Argentina, Brasil, Chile, España, México y Portugal con una producción importante, y Colombia, Cuba, Perú y Venezuela que presentan baja producción en el área. Los investigadores de Iberoamérica han publicado en revistas locales, revistas que no están indexadas en las principales bases de datos internacionales. En estas bases de datos internacionales encontramos, a su vez, pocos textos en español y portugués, revelando así una ausencia de diálogo y accesibilidad entre las búsquedas hechas en bases de datos latinas versus bases de datos anglosajonas.

\section{DISCUSIÓN}

Esta investigación exploratoria resultó compleja y extensa. En el campo de la educación musical no hay estudios comparativos exhaustivos respecto de la situación de la investigación en Iberoamérica, aunque sí encontramos diversos informes acerca del estado de la educación artística en la educación primaria (Giráldez y Palacios 2014) y las políticas en materia de educación artística en Europa (EACEA-EURYDICE 2009), así como diversos trabajos disponibles en la web de la Organización de las Naciones Unidas para la Educación, la Ciencia y la Cultura acerca de la educación artística en cada uno de estos países. Existe una necesidad de revisión de las trayectorias de la investigación en educación musical fuera del mundo anglosajón -cuyo predominio es indiscutible-, con el fin de conocer prácticas relevantes y que tienen un significado para la construcción del campo, como el trabajo coordinado por Terrien y Leroy (2011) en Francia en relación con las perspectivas actuales

8 En Costa Rica se destaca el trabajo de investigación en educación musical del Dr. Guillermo Rosabal Coto (Shifres y Rosabal-Coto 2017). 
en la investigación en educación musical, y también el compendio de artículos organizado y editado por Torres-Santos (2017) acerca de educación musical en el Caribe y América Latina.

Otra característica que hace que este tipo de investigación sea más compleja es el hecho de que no todos los artículos son esencialmente científicos, teniendo en cuenta que una comunicación científica debe regirse, en principio, por una práctica o discurso científico y con un fundamento en estudios científicos anteriores o paralelos, afines a la temática discutida. Muchos artículos del área de la musicología, educación y educación musical encontrados en América Latina son ensayos sin preocupación metodológica o sin referencias. De la misma manera, pueden ser relatos de experiencias individuales o colectivas, o artículos de opinión o reseñas acerca de un único referencial o síntesis temática de un grupo de textos seleccionados sin intención sistemática. Históricamente, no hay una tradición investigadora relacionada con los doctorados a diferencia de lo que sucede en el campo de las ciencias.

Así, hacemos la distinción entre textos académicos científicos y textos académicos informativos no anclados en algún paradigma de la ciencia (Kuhn 2013) o procedimiento metodológico científico. En este contexto es necesario considerar el concepto de lo que es o no es una investigación científica o una obra o un informe científico sobre la base de principios de método y metodología. Para esta consideración científica, propia de la investigación en educación musical, partimos de los trabajos de Díaz Gómez (2006), Díaz Gómez y Giráldez Hayes (2013), Hernández Sampieri, Fernández Collado y Baptista Lucio (2013) y Phillips (2008). De este modo, optamos por hacer una lectura y selección de los trabajos basados en criterios de identificación de artículos que explícitamente comunican investigaciones científicas desarrolladas en centros de posgrado stricto sensu por autores individuales o colectivos pertenecientes al cuadro de estudiantes, profesores o investigadores de las universidades que son reconocidas por los ministerios de educación de cada país. En muchos casos hay artículos que abordan la temática de la educación musical, pero no proceden de estudios académicos sistematizados, provenientes de disertaciones, tesis o de estudios desarrollados por grupos de investigación, o incluso de proyectos individuales de académicos de las universidades.

Otro factor interesante, que hace más complejo este tipo de revisión, es que los autores, investigadores del área, publican en diferentes revistas y en diferentes idiomas. Como la circulación de la producción en esos países vecinos es intensa, resulta extremadamente laborioso separar a autores por nacionalidad, idioma y área de estudio. Es común encontrar al mismo investigador o investigadora publicando en diferentes revistas de diferentes nacionalidades.

También verificamos que es creciente la presencia de nuevos cursos de licenciatura en música en casi todos los países de América Central y América Latina. En ese sentido, encontramos también varias monografías de graduación en licenciatura, sea en pedagogía o área afín como en psicología y en las licenciaturas en música. Asimismo, es posible encontrar muchas publicaciones de las comunicaciones de anales de congresos y eventos del área, principalmente en América Latina donde hay pocos cursos de doctorado con espacio para la investigación en educación musical y pocas revistas que publican artículos del área.

Es muy común en el contexto iberoamericano la investigación acerca de la formación del músico, a causa de la presencia tanto de formación musical profesional en los conservatorios y escuelas de artes como de cursos de música de nivel preuniversitario o técnico-profesional. También es común la presencia de temáticas relacionadas con la enseñanza de música para la formación general del ser humano, principalmente relacionada a fundamentos, metodologías, espacio escolar, currículo y legislación.

En ese sentido, la investigación en Educación Musical en los países iberoamericanos presenta dos características que podemos resaltar. Por un lado, está en desfase en relación 
con las temáticas ya discutidas en la investigación en Educación Musical en los países desarrollados. Es decir, lo que se discute de temáticas actuales en los países europeos y en Norteamérica sigue siendo poco abordado en los países en desarrollo. Por otro lado, se verifica que, de manera semejante a lo que ocurrió hace décadas en los países desarrollados, hay una significativa presencia de temáticas dirigidas a la discusión en torno a la importancia de la música en la formación general del ciudadano, para la formación global e integral del ser y para la inserción de la música en el currículo escolar.

Además, se debe observar la ausencia de publicaciones indexadas en JCR y la escasa presencia en otras bases de datos. Así ya lo señalaban Gustems Carnicer y Calderón Garrido en su estudio bibliométrico, al afirmar que "el escaso número de revistas especializadas y el difícil acceso a las mismas e incluso su desconocimiento, dificultan una mayor difusión de los trabajos realizados" (2014: 28).

Así, la investigación acerca de educación musical, involucrando metodologías, didáctica, legislación, formación de profesores, y de la situación de la enseñanza de música en el contexto de la educación básica, aparece como una temática constante que, en contraste con la situación de desfase de la calidad educativa de estos países, revela una preocupación por hacer más democrático el acceso al contenido y a la formación musical para todos.

Como afirma Palacios Sanz, "la primera reflexión que cabe hacer sería la necesidad de emprender investigaciones similares en nuestro país para replicar los resultados ya conocidos o para cambiar algunas variables y descubrir nuevos efectos. En este sentido, hay muchos caminos abiertos de cara a las futuras investigaciones" (2005: 133).

\section{BIBLIOGRAFÍA}

Abril, Carlos R. y Johanna E. Abril

2017 "Educación Musical Escolar en las Américas: Condiciones, Prácticas, y Políticas desde una Perspectiva Socio-Ecológica”, Revista Electrónica Complutense de Investigación en Educación Musical-RECIEM, 14, pp. 29-46. DOI: 10.5209/RECIEM.57178

Aróstegui, José Luis

2010 "Formación del profesorado de música: Planes de estudio en Europa y América Latina", Profesorado: Revista de currículum y formación del profesorado, XIV/2, pp. 3-7. Disponible en: https://recyt.fecyt.es/index.php/profesorado/issue/view/2352 [acceso: 18 de mayo de 2018].

BALERDi, BEATRIZ

2007 "Bibliografía de investigación musical en Euskal Herria: tesis doctorales", Musiker, 15, pp. 297-326. Disponible en: http://www.eusko-ikaskuntza.eus/es/publicaciones/fondosmusicales-en-bibliotecas-y-archivos-de-euskal-herria/art-17067/ [acceso: 18 de mayo de 2018].

Bordones, MerLina

2013 "Educación Musical en Venezuela”, Revista Ciencias de la Educación, XXIII/41, pp. 119-132.

Bowman, Wayne. D. y Ana Lucia Frega (Eds.)

2012 The Oxford Handbook of Philosophy in Music Education. Oxford: Oxford University Press.

BRASIL

2008 Lei $n^{\circ}$ 11.769, de 18 de agosto de 2008. Altera a Lei no 9.394, de 20 de dezembro de 1996, Lei de Diretrizes e Bases da Educação. Disponible en: http:/ / www.planalto.gov.br/ccivil_03/_Ato20072010/2008/Lei/L11769.htm [acceso: 20 de mayo de 2018].

Bray, Mark y Bob Adamson

2010 Educación comparada: enfoques y métodos. Buenos Aires: Ediciones Granica. 
Burgos Peredo, Óscar Eduardo, Francisco Javier Perales Palacios y José Gutiérrez Pérez 2010 "Evaluación de la calidad de los establecimientos educativos incorporados al sistema nacional de certificación ambiental de la provincia de Bio Bio (Chile)“, Profesorado: Revista de currículum y formación del profesorado, XIV/2, pp. 213-240. Disponible en: https:/ / recyt.fecyt. es/index.php/profesorado/article/view/42910 [acceso: 18 de mayo de 2018].

Capistrán Gracia, Raúl W.

2021 “Qué es lo que queremos de la educación musical?”, La educación musical en el nivel básico en México, retos y propuestas actuales. Luis Alfonso Estrada Rodríguez, Laura Elizabeth Gutiérrez Gallardo, Federico Sastré Barragán (editores). Ciudad de México: UNAM/Facultad de Música, pp. 102-120.

Cateura Mateu, Maria.

1992 Por una educación musical en España: estudio comparativo con otros países. Barcelona: PPU.

Cykler, Edmund A.

1969 "Comparative Music Education", Journal of Research in Music Education, XVII/1, pp. 9-16. DOI: $10.2307 / 3344202$

Díaz Gómez, Maravillas (Coord.)

2006 Introducción a la investigación en educación musical. Madrid: Enclave Creativa.

Díaz Gómez, Maravillas y Andrea Giráldez Hayes (Coords.)

2007 Aportaciones teóricas y metodológicas a la educación musical. Barcelona: Graó.

2013 Investigación cualitativa en educación musical. Barcelona: Graó.

EACEA-Euridice

2009 Educación artística y cultural en el contexto escolar en Europa. Disponible en: https://sede.educacion.gob.es/publiventa/educacion-artistica-y-cultural-en-el-contexto-escolar-en-europa/ educacion-estadisticas-union-europea-ensenanzas-artisticas/13906 [acceso: 19 de mayo de $2018]$.

Fernandes, José N.

2014 "Educação musical internacional e comparada: uma introdução". Debates - UNIRIO, 13, pp. 51-58. Disponible en: http://www.seer.unirio.br/index.php/revistadebates/article/ view/4614 [acceso: 18 de mayo de 2018].

Flinders, David J. y Carol P. Richardson

2002 "Contemporary issues in qualitative research and music education", The New Handbook of Research on Music Teaching and Learning. Richard Colwell y Carol Richardson (editores). Nueva York: Oxford University Press. pp. 1159-1175.

Gillanders, Carol y Pedro Martínez Casillas

2005 "La investigación en el ámbito musical", Música y Educación, XVIII/64, pp. 85-104.

Giráldez Hayes, Andrea y Andrés Palacios

2014 Educación artística en Iberoamérica: Educación primaria. Madrid: OEI.

Goergen, Pedro L.

1991 "Educação comparada a uma disciplina atual ou obsoleta?”, Pro-posições, II/3, pp. 12-23.

Green, LuCY.

1997 Music, Gender, Education. Cambridge: Cambridge University Press.

Gustems Carnicer, Josep y Diego Calderón Garrido

2014 "Estudio bibliométrico de los artículos de educación musical incluidos en DIALNET 20032013”, Revista Electrónica de LEEME. Lista Electrónica Europea de Música en la Educación, 33, pp. 27-39. Disponible en: https://ojs.uv.es/index.php/LEEME/issue/view/744 [acceso: 18 de mayo de 2018]. 
Gutiérrez GonzÁlez, José M.

2011 "La formación inicial del profesorado de secundaria. Del CAP al Máster", CEE Participación educativa, 17, pp. 96-107.

Hanley, Betty y Janet Montgomery

2002 "Contemporary curriculum practices and their theoretical bases", The New Handbook of Research on Music Teaching and Learning. Richard Colwell y Carol Richardson (editores). Nueva York: Oxford University Press. pp.113-143.

Hemsy de Gaínza, Violeta

1998 "La educación musical en los tiempos ecológicos", La nueva musicoterapia. Rolando Benenzon, Violeta Hemsy de Gaínza y Gabriela Wagner (autores). Buenos Aires: Lumen. pp. 153-158.

2004 "La educación musical en el siglo XX". Revista Musical Chilena, LVIII/201, pp. 74-81. Disponible en: https://revistamusicalchilena.uchile.cl/index.php/RMCH/article/ view/12449_[acceso: 18 de mayo de 2018].

2010 "Educação musical e contemporaneidade (Entrevista por Elízabeth Carrascosa Martínez)", Revista Espaço Intermediário, I/2, pp. 12-15. (Edición limitada).

Hernández Sampieri, Roberto, Carlos Fernández Collado y María del Pilar Baptista lucio 2013 Metodologia de Pesquisa. Coleção métodos de pesquisa (5 $5^{\mathrm{a}} \mathrm{ed}$.). Porto Alegre: Penso (Mc Graw Hill).

IRWIN, RITA

2008 "A/r/tography as practice-based research", Arts-based Research in Education. Melisa CahnmannTaylor y Richard Siegesmund (editors). Nueva York: Routledge. pp.103-124.

Jiménez Pérez, Miguel A.

2015 "La Allemande Tombeau de Mr.Françque de Robert de Viseé y el proceso de su transcripción para guitarra. Un ejemplo de investigación desde la interpretación”, Enseñar Música: Revista Panamericana de Investigación, III/1, pp. 31-41.

Kemp, Anthony E. (ED.)

1993 Aproximaciones a la investigación en educación musical (título original: Some Approaches to Research in Music Education, 1992). Ana Lucía Frega y Dina Poch de Grätzer (traductoras). Buenos Aires: Collegium Musicum.

Kemp, ANTHONy y LAURence LePHerd

1992 "Research methods in international and comparative music education", Handbook of Research in Music Teaching and Learning. Richard Colwell (editor). Nueva York: Schirmer Books, pp.773-788.

Kunn, Thomas S.

2013 A estrutura das revoluções científicas (12ª ed). São Paulo: Perspectiva.

LARRAÑaga TORRóNTEgui, RAMóN

2016 Educación musical escolarizada en México 1920-1940. Disponible en: http://www.ilustrados. com/tema/6806/Educacion-musical-escolarizada-Mexico-1920-1940.html [acceso: 19 de mayo de 2018].

LeVitin, DANiEL J.

2018 Tu cerebro y la música: Por qué nos gusta la música y por qué disfrutamos con ella. José Manuel Álvarez-Flórez (traductor). Barcelona: RBA Bolsillo.

Luna Ortiz, Roberto

2008 "Educación musical en la escuela primaria de tiempo completo". Tesina. México. Disponible en: 200.23.113.51/pdf/25165.pdf [acceso: 20 de mayo de 2018].

Margaño Mena, Luis. ET AL.

1961 "Informe del comité de profesores de educación secundaria". Primer congreso de educación musical Chile - 1958. pp. 107-103. Disponible en: http://www.revistamusicalchilena.uchile. cl/index.php/RMCH/article/viewFile/16088/16603 [acceso: 19 de mayo de 2018]. 
Marín Viadel, Ricardo, José Luis Tolosa Marín y Juan Fernando de Laiglesia

1998 La investigación en Bellas Artes. Tres aproximaciones a un debate. Granada: Grupo Editorial Universitario.

Mark, Michael L.

1992 "A history of music education research", Handbook of Research on Music Teaching and Learning. Richard Colwell (editor). Nueva York: Schirmer Books. pp.48-59.

Martínez Rivera, Victor S.

2016 "Retos para la educación musical en México", Correo del Maestro-Revista para professores de educación básica. Disponible en: http:/ /www.correodelmaestro.com/publico/html5032014/ capitulo5/capitulo_05.html [acceso: 19 de mayo de 2018].

México. Secretaría de Educación Pública

2011a Programas de estudio 2011, guía para el maestro. Educación Básica. Secundaria. Artes. México: SEP.

2011b Programas de estudio 2011, guía para el maestro. Educación Básica. Primaria. Primer grado. México: SEP.

Mota, Graça

2014 "A educação musical em Portugal - uma história plena de contradições", DEBATES - Cadernos do Programa de Pós-Graduação em Música, 13, pp. 41-50.

Narejos, Antonio

2017 Tesis doctorales de música en España. Base de datos online. Disponible en: http://www.tesisdemusica.es/ [acceso: 18 de mayo de 2018].

Oriol de Alarcón, Nicolás

2002 "El área de <Didáctica de la Expresión Musical>, Revista de Educación, 338, pp. 155-166.

2007 "Enseñanza musical en España”, Aportaciones teóricas y metodológicas a la educación musical. Maravillas Díaz Gómez y Andrea Giráldez Hayes (coordinadoras). Barcelona: Graó. pp. 87-94.

2009 "La investigación musical en España: tesis doctorales y temática en la última década”, Eufonía, 45, pp. 59-87.

2012 “Temáticas de tesis doctorales de Música en España (1978-2011)”, Música y Educación, XXV/92, pp. 58-94.

Palacios Sanz, José Ignacio

2005 "La Universidad y la investigación musical: de la teoría a la praxis". Revista Interuniversitaria de Formación del Profesorado, XIX/52, pp. 123-156.

Palheiros, Graça B.

1999 "Investigação em educação musical: perspectivas para o seu desenvolvimento em Portugal", Revista Música, Psicologia e Educação, 1, pp. 15-26.

Pastor Gordero, Pasqual.

2002 "La investigación educativa musical”, Eufonía, 26, pp. 84-88.

Pereira, Eliton Perpetuo Rosa

2019 "A Educação Musical no Brasil: temáticas, concepções e linhas investigativas". Tesis doctoral en educación. Universidad de Santiago de Compostela. Santiago de Compostela. Disponible en: https://minerva.usc.es/xmlui/handle/10347/20495 [acceso: 30 de noviembre de 2019].

Phelps, Roger P., Lawrence Ferrara y Thomas W. Goolsby

1993 A Guide to Research in Music Education. Londres: The Scarecrow Press.

Phillips, Kenneth H.

2008 Exploring Research in Music Education E $\mathcal{F}^{2}$ Music Therapy. Nueva York: Oxford University Press.

Poblete lagos, Carlos

2010 "Enseñanza musical en Chile: continuidades y cambios en tres reformas curriculares (1965, 1981, 1996-1998)", Revista Musical Chilena, LXIV/214, pp. 12-35. 
2017 "Formación docente en música en Chile: Una aproximación histórica desde tres universidades", Revista da FAEEBA -Educação e Contemporaneidade. XXVI/48, pp. 97-109. DOI: 10.21879/ faeeba2358-0194.2017.v26.n48.p97-109

Poblete lagos, Carlos, Adrián Leguina, Nicolás Masquiarán y Bárbara Carreño

2019 "Informal and non formal music experience: power, knowledge and learning in music teacher education in Chile”, International Journal of Music Education, XXXVII/2, pp. 272-285. DOI: $10.1177 / 0255761419836015$

Porta Navarro, Amparo

2014 "La Educación Musical y su difusión científica. Una forma de aproximación a través del análisis de contenido", Actas III Congreso CEIMUS. Enseñary aprender Música. Modelo tradicional, online y semipresencial. Madrid: Enclave Creativa, pp. 605-613.

Real Academia Española

2018 Iberoamérica. Disponible en: http://buscon.rae.es/dpd/srv/search?id=qDQJAJJhCD6g22Go4k [acceso: 20 de mayo de 2018].

Regelski, Thomas A.

1975 Principles and problems in music education. Nueva Jersey: Prentice-Hall

REIMER, BENNETT.

2006 "Toward a Philosophical Foundation for Music Education Research", MENC Handbook of Research Methodologies. Richard Colwell (editor). Nueva York: Oxford University Press. pp. 3-37.

Rodriguez Melo, Martha E.

2015 "Educación musical en Colombia: Entre el impulso a debilitar y el impulso a ampliar". XXII Congresso nacional de Associação Brasileira de Educação Musical ABEM. Anais da ABEM. Natal $R N$, Brasil. Disponible en: http://abemeducacaomusical.com.br/conferencias/index.php/ xxiicongresso/ [Acceso: 30 de junio de 2017].

RumpF, RANDY J. J.

2012 "A systematic review of music teacher education research within the United States: 19822010". Tesis doctoral. Universidad de Maryland. Disponible en: https://drum.lib.umd.edu/ handle/1903/13192 [acceso: 20 de mayo de 2018].

RUSINEK, GABRIEL

2007 "Tradition and change in the Spanish Music Curriculum", International Handbook of Research in Arts Education. Liora Bresler (editora). Dordrecht: Springer, pp. 173-174.

Shifres, Favio y Guillermo Rosabal-Coto

2017 "Hacia una educación musical decolonial en y desde Latinoamérica", Revista Internacional de Educación Musical, 5, pp. 85-91. DOI: 10.12967/RIEM-2017-5-p085-091

Siegel, DANiel J.

2007 The Mindful Brain: Reflection and Attunement in the Cultivation of Well-Being. Nueva York: WW Norton.

Subirats Bayego, Maria dels A.

2011 "La investigación en Didáctica de la Expresión Musical", Educatio Siglo XXI, XXIX/1, pp. 175194. Disponible en: https://revistas.um.es/educatio/article/view/119931 [acceso: 18 de mayo de 2018].

Teachout, David James

2012 "The preparation of music teacher educators: a critical link", The Oxford Handbook of Music Education (2a ed.), vol. 2. Gary E. McPherson y Graham F. Welch (editores). Nueva York: Oxford University Press, pp. 685-688.

Terrien, Pascoal y JeAn-Luc Leroy

2011 Perspectives actuelles de la recherche en éducation musicale. París: L'Harmattan.

Thayer Sataloff, Robert, Alice G. Brandfonbrener y Richard J. Lederman

1998 Performing Arts Medicine. California: Singular Publishing Group. 
TORRES-SANTOS, RAYMOND (EDITOR)

2017 Music Education in the Caribbean and Latin America: A comprehensive guide. Londres: Rowman \& Littlefield.

Vélez-Cuartas, Gabriel, Diana Lucio-Arias y Loet LeydesdorfF

2015 "Regional and global science: Publications from Latin America and the Caribbean in the Scielo Citation Index and the Web of Science", El profesional de la información, XXV/1, pp. 35-46. Disponible en: http://www.elprofesionaldelainformacion.com/contenidos/2016/ ene/05.pdf [acceso: 18 de mayo de 2018].

Volk, TERESE M.

1997 Music, Education and Multiculturalism. Oxford: Oxford University Press. 